\title{
Zur Behandlung der exsudativ-lymphatischen Diathese.
}

\author{
Von \\ Prof. I). S. MONRAD, Copenhagen. \\ Vortrag am 2. Nordischen Kongress für Padiatrie, \\ Stockholm 19.-21. Juni 1921. \\ Meine Damen und Herren!
}

Da die exsudative oder - wie ich zu sagen vorziehe die exsudativ-lymphatische Diathese ohne Zweifel bei unserem nächsten Kongress ein Hauptthema bilden wird, mag es vielleicht wenig angebracht erscheinen, dass ich veranlasst habe, dass die Frage bereits heuer auf die Tagesordnung gestellt worden ist. Wenn ich dies dennoch getan habe, so ist es auch keineswegs geschehen, um die künftige Generaldebatte über diese wichtige und eigentümliche Konstitutionsanomalie irgendwie zu antizipieren, sondern nur weil ich es als opportun betrachte, Sie bereits jetzt mit den therapeutischen Versuchen bekannt zu machen, die ich in den letzten Jahren angestellt habe, und zwar in der Hoffnung, dass einige meiner werten Kollegen dieselben vor dem nächsten Kongress nachprüfen möchten.

Ich glaube, dass wir schnell darüber einig werden können, dass unsere Kenntnis des innersten Wesens dex exsudativlymphatischen Diathese eine äusserst mangelhafte ist, indem die verschiedenen Stoffwechselversuche, Blutuntersuchungen sowie die sonstigen Forschungsresultate bislang teilweise einander ganz widerstrebende Resultate ergeben haben. Dass eine der- 
artige Unsicherheit in betreft der Pathogenese notwendigerweise der Behandlung ein unsicher tastendes Gepräge verleihen muss, liegt auf der Hand, und wir sehen denn auch die verschiedenen Verfasser je ihren therapeutischen Weg einschlagen. So verbietet Cnwn Rahm, Eier und Zucker, gestattet aber ein wenig Butter und Milch sowie Fleisch und rohes Obst. SAlge ist gleichfalls gegen Eier und Mich, warnt aber zugleich vor Fleisch. Phivinuse verbietet Eier, Zncker, Butter und Rahm, erlaubt aber bis $1 / 2$ 1. Milch pro Tag. Und schliesslich behauptet Nrwasxs, Rahm und Butter seien ganz unschädlich und die Gefahr bei reichlicher Zufuhr von Kuhmilch bei exsudativen Kindern liege nicht am Milchfett, sondern an den Eiweissstoffen der Milch. Wir finden hier also etwas für jeden Geschmack.

In einem Punkt scheinen die meisten Verfasser jedoch ibereinzustimmen, nïmlich in betreff des schädlichen EinHusses der Überenährung auf diese Kinder, und daher lautet iberall die Parole, dass die Hauptsache bei der Behandlung. in der Termeidung jeglicher Form von Müstung bestehe. CzERNy sagt ja auch an einer Stelle, dass es darauf ankomme, diesen Kindern nur weniger Kalorien pro Kilogramm und 'Гам' zuzuführen, als sonst erforderlich.

Dass diese Betrachtung allgemeinen Anschluss hat finden können, beruht sicherlich auf dem Umstande, dass man sich viel zu einseitig mit den exsudativ-lymphatischen Kindern des fetten, pastösen Typus beschäftigt und den magern, erethischen Typus nicht hinlänglich berücksichtigt hat, und zwar trotzdem der magere Typus weit häufiger vorkommt als der fette, jedenfalls wenn von über 2 Jahre alten Kindern die Rede ist.

Seit einer Reihe von Jahren habe ich nun bei der exsudativ-lymphatischen Diathese zahlreiche Fütterungsversuche angestellt und bin dadurch zu dem Resultat gekommen, dass das entscheidende Moment der Behandlung nicht in der grösseren oder geringeren Anzahl von zugeführten Kalorien, sondern in der Art der Nahrung zu suchen ist. Bei diesen Versuchen war meine Aufmerksamkeit in ersten Reihe auf Kinder des magern Typus gerichtet. Diese Kinder werden Sie alle 
aus Ihrer Konsultationspraxis kennen: magere, schlaffe, mïde, pseudoanämische, oft subfebrile Kinder mit fortwährend rezidivierenden Rhino-Tracheo-Bronchitiden, mit Hyperplasie aller drei Tonsillen und mit periodischen Ausbrüchen von Strophulus und Seborrhoea, Kinder, die meist lange Zeit hindurch mit Eiern, Butter und Schlagrahm gefüttert wurden, deren exsudativ-lymphatische Symptome sich aber bei dieser Behandlung unverändert erhalten oder gar verschlimmert haben, und die (trotz der stärkenden und mästenden" Kost) fortgesetzt müde, schlaff und mager sind - sie verhalten sich tatsïchlich wie Pharaos magere Kühe.

Bei solchen Kindern habe ich nun einmal ums andere die Erfahrung gemacht, dass man bei Entfernung von jeglichem tierischen lett aus der Kost und ausschliesslicher Verabreichung. von Eiweissstoffen, Kohlenhydraten und Pflanzenfett die exsudativ-lymphatischen Symptome schwinden, das Allgemeinbefinden sich bessern und das Gewicht zunehmen sieht. Diese Gewichtzunahme rührt nicht von lose gebundenem Wasser her, ist vielmehr eine solide, andauernde Zumahme, während welcher das Fettgewebe fest, die Muskulatur kräftiger und der Turgor ein guter wird.

Wende ich dasselbe diatetische Verfahren bei Kindern des fetten, pastösen Typus an, so sehe ich, wie das Fettgewebe dieser Kinder gleichsam ungestimmt wird; die Kinder nehmen nicht besonders stark an Gewicht ab - was ich auch nicht anstrebe - , ihr Fettgewebe aber, das zuvor lose; pastös und ödematös war, wird nun fest und der Turgor normal, bei gleichzeitigem Schwinden der verschiedenen Exsudationen an Haut und Schleimhäuten.

Die Geschwindigkeit, mit der sich die Wirkung dieser diatetischen Behandlung zu erkennen gibt, ist sehr verschieden. So ist die Wirkung bei einigen Kindern bereits nach einer Behandlung von einigen Wochen unverkennbar, bei anderen Kindern können aber Monate verstreichen, bevor man eine wirkliche Besserung erkennt.

Auch was die verschiedenen Symptome betrifft, macht sich ein grosser Unterschied bemerkbar, indem die Exsuda19-.-212as. Acta padiatrica. Vol. I. 
tionen an Haut und Schleimhäuten weit schneller und früher schwinden als die Hyperplasie der lymphoiden Gefässe. Während man Strophulus, Ekzeme und Bronchitiden im Laufe von wenigen Wochen zum Schwinden bringen kann, dauert es oft mehrere Monate, bevor adenoide Vegetationen und hypertrophische Tonsillen in nennenswertem Grade an Grösse abnehmen.

Schliesslich ist es ron Wichtigkeit hervorzuheben, dass man die diätetische Behandlung nicht gleich aufoeben darf, sobald die Symptome geschwunden sind, denn in diesem Falle würden sich leicht Rückfälle ergeben. Man muss lange Zeit hindurch, oft Monate oder halbe Jahre lang mit der Diät fortfahren, nachdem die letzten Symptome sich verloren haben, und man kann es auf diese Weise auch dazu bringen, dass die Diathese latent bleibt.

Dass man bei hinreichend langer, konsequent durchgeführter diätetischer Behandlung auch eine radikale Genesung erzielen kann, betrachte ich als zweifellos; es ist aber schwer, zu entscheiden, inwiefern die Genesung im Einzelfalle von der Behandlung herrïhrt, da die Diathese ja mit den Jahren spontan schwindet.

Die von mir benutzte, auf empirischem Wege ausfindig. gemachte Behandlung besteht also, wie ich Ihnen eben sagte, darin, dass ich die Verabreichung von tierischem Fett auf ein Kinimum reduziere oder dasselbe ganz aus der Kost entferne.

Kinder unter 1 Jahre erhalten also weder Frauenmilch noch gewöhnliche Vollmilch, jedenfalls nur sehr kleine Mengen, dagegen Magermilch (entweder als Milchgemisch oder als Brei) und Buttermilchsuppe; später aufgeweichten Zwieback, Hafersuppe, Saftsuppe, Kartoffelmus, Apfelmus u. dgr.

Für grössere Kinder verbiete ich: Vollmilch, Rahm, Butter, Schmalz, Eidotter und Speck sowie fette Fleisch- und Fischsorten, während jegliche sonstige Nahrung gestattet wird, und der Speisezettel dieser Kinder hat dann in der Hauptsache folgendes Aussehen: Milchspeisen, hergestellt aus Magermilch, Buttermilchsuppe, in Wasser gekochte Grütze, Hafergxütze, Graupensuppen, Fleischsuppe, Brot, Gemüse und Kartoffeln, 
Obst und allerlei Obstgerichte, Honig und Marmelade; fermer Eiweiss, mageres Kalbs- und Rindfleisch sowie Dorsch und Schollen, und, da Pflanzenfett keinen schädlichen Einfluss ausübt, dürfen diese Kinder auch Pflanzenbutter, Pflanzenmargarine, Kakao und milchfreie Chokolade erhalten.

Man hat also, wie Sie sehen, Gerichte genug zur Verfïgung, und wenn es wegen Anorexie oder anderer Verhältnisse schwer fällt, eine hinlängliche Zufuhr von Kalorien $z u$ erzielen, pflege ich Malzextrakt, $20-50 \mathrm{~g}$ tägl., vorzuschreiben. Dagregen rate ich Lebertran $a b$, den ich früher benutate, in den letzten Jahren aber ganz aufgegeben habe, da ich von ihm ebenso schädliche Wirkungen wie von allen anderen tierischen Fetten gesehen zu haben meine.

Wie streng die hier skizzierte Diät durchgeführt werden soll, hängt bis zu einem gewissen Grad vom Charakter des vorliegenden Falles ab. Es gibt einzelne exsudativ-lymphatische Kinder, denen man ohne Schaden kleinere Mengen von tierischen Fett geben kann, anderseits trifft man aber viele Kinder, bei denen sogar minimale Mengen von tierischem Fett schädlich wirken. Dessen hat man sich namentlich zu erinnern, wenn es sich um Kinder handelt, die zu Hause behandelt.werden, wo es - gans natürlich - schwer fällt, zu verstehen und daran zu glauben, dass ein wenig Fettstoft Schaden anrichten kann. Ich kann Ihnen hier ein instruktives Beispiel aus meiner privaten Praxis anführen. Es handelte sich um ein 6-jähriges Mädchen, das mehrere Jahre hindurch stark ausgesprochene exsudativ-lymphatische Symptome (Strophulus, adenoide Vegetationen, Tonsillenhyperplasie, Bronchitiden usw.) aufgewiesen hatte, und bei dem die vorgeschriebene Diät zu meinem Erstaunen nicht wirkte. Ich brachte das Kind dann im Krankenhause unter und erzielte nun nach ein paar Monate lang streng durchgeführter Diät eine eklatante Wirkung. Da ich nun die Verhältnisse näher untersuchte, zeigte es sich, dass das Hausfräulein - dem gegebenen Auftrag zuwider und ohne dass die Mutter davon wusste - den Nachmittagskakao des Kindes mit Vollmilch statt mit Magermilch zubereitet hatte, und diese tägliche Zufuhr 
von etwa ${ }^{1}$ is 1 . Vollmilch hatte also genügt, um die Diathese aufrecht zu erhalten.

Die kurativen Wirkungen der angefiihrten Diät bei der exsudativ-lymphatischen Diathese habe ich nun so oft ror mir grehabt, dass es ganz ausgeschlossen ist, dass hier von Zufälligkeiten die Rede sein kann. Ich verfüge nämlich über ein recht ansehnliches Material. So habe ich allein in den letzten 4 Jahren, 1917-1920 einschliesslich, Gelegenheit gehabt, 327 exsudativ-lymphatische Kinder zu behandeln, von denen 124 in Dronning Lonises Bornehospital untergebracht waren und 203 zu meiner privaten Klientel gehörten.

Damit Sie selbst den Wert meiner Beobachtungen ein. schätzen können, werde ich mir gestatten, Ihnen in aller Kürze einige meiner typischsten Fälle mitzuteilen.

I. 3-jähriger Knabe wegen Ekzem im Krankenhaus untergebracht. Litt schon seit dem Säuglingsalter an einem bald nässenden, bald trockenen Ekzem des Haarbodens sowie an trocknem, schuppendem Ekzem am Truncus. Wurde lange Zeit hindurch von einem dermatologischen Spezialisten mit verschiedenen Salben und Pasten behandelt, jedoch ohne Erfolg. War 3 Monate mit Muttermilch, später mit Griessbrei und sonstiger Flaschenkindkost genährt worden. Seit den letzten Monaten bedeutende Verschlimmerung des Ekzems bei lakto-vegetabilischer Kost, reichlich ergänzt mit Rahm und Butter.

Status praesens bei der Aufnahme: pastös (etwa $2 \mathrm{~kg}$. U̇bergewicht), muskelschlaff, pseudoanämisch; der ganze Haarboden wund von teils nässendem, teils trocknem Ekzem; am Truncus ausgebreitete, trockne, schuppende Dermatitis. Ausserdem: Blepharo-Conjunctivitis und Tonsillenhyperplasie.

Nach 2 monatl. Behandlung mit Diat (und Pellidolsalbe) waren die Ekzeme vollständig geschwunden.

Das Kind wurde nach 1 Jahre wieder vorgestellt: die Haut sauber; die Tonsillen kleiner.

II. S-jähriges Mädchen konsultierte mich wegen fortwährend rezidizierenden Bronchitiden mit asthmatischen Anfälen.

Ein Bruder hat an infantilem Ekzem und Strophulus 
gelitten. Das Mädchen selbst hat in der Dentitionsperiode Strophulus gehabt und, seit dem 2. Lebensjahre, zahlreiche Bronchitiden mit periodischen Anfällen von Asthma. Im Alter von 3 Jahren war Adenotomie und im Alter von 5 Jahren wiederum Adenotomie nebst Tonsillotomia duplex vorgenommen worden.

Status praesens: diffuse Bronchitis, Tonsillenhyperplasie, adenoide Vegetationen und Strophulus.

Nach 3 monatl. diatetischer Behandlung schrieb mir die Mutter: sist jetzt ein ganz anderes Kind".

III. 10-jährige Tochter eines Arates konsultierte mich wegen ehronischer Bronchitis mit Asthma. Wurde vor 4 Jahren adenotomiert und tonsillotomiert und ist fortwihrend mit $\mathrm{E} i$ dottern und Schlagram genährt worden. Ist inüde, mager und pseudoanämisch, hat Strophulus, Intertrigo and Seborrhoea nebst fortwährend rezidivierenden Bronchitiden und Asthma.

Nach $1 / 2$ jähr. diätetischer Behandlung haben die Bronchitiden ganz aufgehört; sie hat nur selten asthmatische Anfülle und hat nur ein paar vereinzelte Ausbrüche von Strophulus wehabt. Sieht frischer aus und hat $4 \mathrm{~kg}$ zugenommen.

IV, 8-jähriges Mädchen aus Jïtland, im Kinderkrankenhans untergebracht wegen Bronchitis und tsthma, welche Leiden seit dem 1. Lebensjahre gedauert und jeder Behandlung getrotzt hatten; ist auch mehrmals im Provinziallionkenhause behandelt worden. - Hatte während der letzten Jahre zahlreiche asthmatische Anfälle und ist bei reichlicher Ernährung mit Rahm und Eievn abgemagert.

Status praesens bei der Aufnahme: ist mager, Untergewicht von etwa $9 \mathrm{~kg}$, blass und mitgenommen; hat diffuse Bronchitis und Emphysema pulmonum mit periodischen Anfällen von Asthma; ferner Lingua geographica, Tonsillenhyperplasie, adenoide Vegetationen und Polyadenitis nebst Hosinophilie (11\%), Lymphocytose (60\%) und Hypoleukocytose (30 \%).

Nach 4 monatl. diätetischer Behandlung wurde eine bedeutende Besserung sämtlicher Symptome und gleichzeitige Zunahme des Gewichtes festgestellt (siehe Kurve I). 
V. Sjähriges Mädchen, Tochter eines Arztes, konsultierte mich im Herbst 1920, da ein interner Spezialist die Diagnose Bronchialdrisentuberkulose gestellt und zu einem Sanatorienaufenthalt geraten hatte.

Leidet seit den ersten Lebensjahren an Strophulus und Seborrhoea und hat in den späteren Jahren an fortwährend rezidivierender Rhino-Tracheo-Bronchitis nebst subfebrilen Temperaturen, Müdigkeit, Abmagerung und Blässe gelitten; hat daher jeden Winter viele Monate lang die Schule versäumen müssen. Ist mit Schlagrahm, Eiern und viel Butter. ernährt worden.

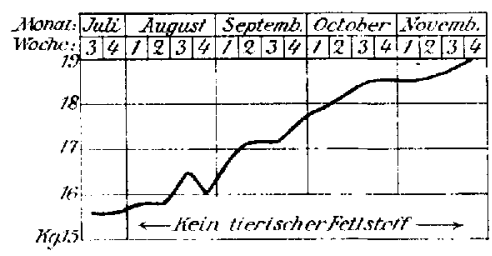

Kirve 1: 8-jähriges Mädehen.

Status praesens: Mager, pseudoanämisch (Hgb 90), Seborrhoea am Haarboden, Tonsillenhypertrophie, diffuse Bronchitis. Tuberkulinprobe negativ.

"Tch diagnostizierte: exsudativ-lymphatische Diathese und beseitigte daher alles tierische Fett aus der Kost - mit dem Ergebnis, dass bereits nach 14 Tagen ein unverkennbarer Unschlag im Zustande des Mädchens zu merken war. Wurde mir im Mai dieses Jahres wieder vorgeführt und sah nun ganz wohl aus, hatte an Gewicht zugenommen und die Schule diesen Winter keinen einzigen Tag wegen Erkältung versäumen müssen. Die Hautaffektionen fast geschwunden, in den Pulmones nichts zu konstatieren und die Tonsillengeschwulst merkbar verkleinert.

IV. 4 3/4-jähriges Mädchen, im Krankenhaus unter der Diagnose: Febris continua (obs. p. tb.) untergebracht. Hatte als Säugling Gneiss. Vom Alter von $1 / 2 \mathrm{Jahr}$ an: wiederholte 
Ausbrüche von Strophulus. Vom Alter von $1 \frac{1}{9}$ Jahren an: wiederholte Anfälle von Rhinitis, Bronchitis und Angina.

In Alter von $2^{1 / 2}$ Jahren wurde Adenotomie und $T_{0, n}$ sillotomia duplex vorgenommen, aber ohne Erfolg.

War jetzt seit mehreren Monaten zu Hause bettlägerig gewesen mit subfebrilen Temperaturen und Bronchitis.

Status praesens bei der Aufnahme: pastös, pseudoanïmisch und müde mit Hyperplasie aller drei Tonsillen, ausgebreiteter Bronchitis, Polyadenitis, Milzgeschwulst, Ekzema intertriginosum hinter den Ohren, und Eosinophilie (15\%). Tuberkulinprobe negativ.

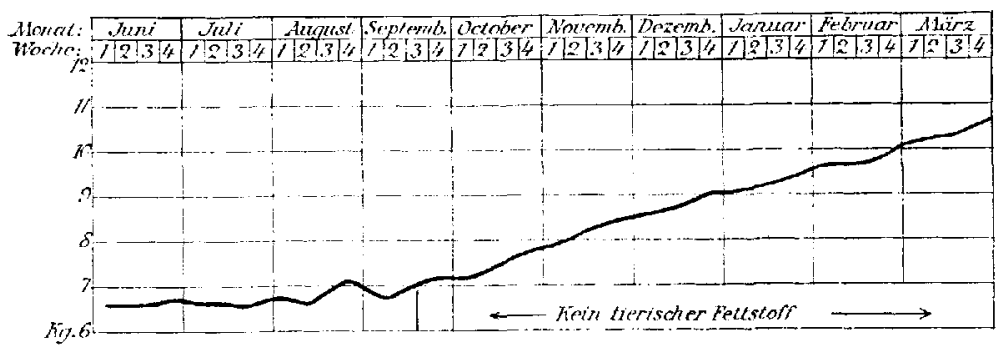

Kurve II: 1-jälıriger Knabe.

Nach 2 monatl. diätetischer Behandlung schwanden sämtliche Symptome, besserte sich das Allgemeinbefinden und nahm das Gewicht zu.

Wurde 1 Jahr später wieder vorgestellt und war gesund.

VII. 3/4-jähriger Knabe wurde im Mai im Kinderkrankenhause untergebracht und mehrere Monate lang wegen Rachitis nebst häufig rezidivierenden Rhino-Tracheo-Bronchitiden behandelt, jedoch ohne Erfolg. Aus irgend einem unerklärlichen Grunde sahen wir erst im September ein, dass der Junge ausgesprochen exsudativ-lymphatisch war, indem er ausser an den genannten Schleimhantleiden an Seborrhoea am Haarboden, Strophulus, Landkartenzunge, Tonsillenhyperplasie, adenoiden Vegetationen nebst Eosinophilie, Lymphocytose und Hypolenkocytose litt. 
Wir entfernten num jeglichen tierischen Fettstoff aus der Kost, was bewirkte, dass sümtliche exsudativ-lymphatischen Symptome im Laufe des Winters stark zurückgingen und das Gewicht gleichzeitig zumahm (siehe Kurve II).

VIII. Die 3. Kurve betrifft einen 9-jühigen Kuaben, der in seinem 1. Lebensjahr ein hartniickiges, typisches infantiles Ekzem hatte und in den späteren Jahren häufig Anfille ron Bronchitis mit Asthma gehabt und viel an seborrhoischem Hkzem gelitten hat.

Status praesens bei der Aufnahme: mager (Untergewicht von $2 \mathrm{~kg}$ ) mit schlechter Muskulatur; Seborrhoea am Haarboden, universelle ichtyosisähnliche Hautaffektion, Tonsillenhypertrophie, diffuse Bronchitis mit Emphysem, Eosinophilie. Tuberkulinprobe negativ.

Wurde von August bis November mit gutem Erfolg mit fettfreier Diät behandelt; wurde darauf in unser Landkrankenhaus gebracht, wo ihm durch ein Versehen gewöhnliche Kost gereicht wurde, d. h. er bekam auch Vollmilch und Butter; er nahm hier an Gewicht ab, und gleichzeitig verschlimmerten sich sämtliche exsudativ-lymphatischen Symptome. - Wurde wieder ins Hauptkrankenhaus gebracht, wo ihm fettfreie Diait verabreicht wurde - und zwar mit der Wirkung, dass die Symptome schwanden und das Gewicht zunahm. Während dieser Periode wurde ihm - experimenti causa - ein par Wochen lang täglich 1 del. Rahm verabreicht, was bewirkte, dass das Gewicht abnahm und die Hautaffektionen sich gleichzeitig verschlimmerten. Nachdem der Rahm ausgeschaltet worden war, besserten sich sämtliche exsudativ-lymphatischen Symptome wiederum und das Gewicht nahm wieder zu.

Ich will Sie nun nicht weiter mit Krankengeschichten ermüden, meine aber, dass die angeführten genügen, um zu zeigen, dass die Materia peceans sicherlich in den tierischen F'ettstoffen der Nahrung zu suchen ist. Es ist bei der Behandlung der exsudativ-lymphatischen Diathese nicht die Mästung an und für sich, die eine Rolle spielt, und man kann in der Tat diesen Kindern fast ebenso viele Eiweissstoffe, Kohlenhydrate und Pflanzenfettstoffe verabreichen, wie man will, 
wenn man nur wit der Zufuhr von animalischem Fett rorsichtig ist.

Durch mehrere meiner Krankengeschichten erhält man den Eindruck, dass die tierischen Fettstoffe wie ein Gift auf die exsudativ-lymphatischen Kinder wirken, und falls meine Beobachtungen und Erfahrungen richtig sind, werden sie vielleicht einen Fingerzeig abgeben können, in welcher Rich-

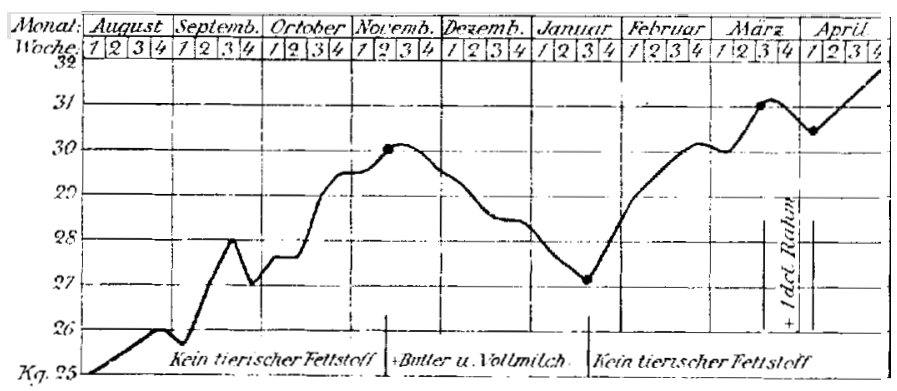

Kutve IIT: 9-jühriger Knabe.

tung die experimentelle Forschung der Zuknnft sich zu bewegen haben wird. Forläufig liegt alles im ungewissen; falls aber die Untersuchungen, die im "Dronning Louises Bornehospital» in Verbindung mit dem Physiologischen Laboratorium der Universität Copenhagen in Angriff genommen worden sind und iber die ich mich im gegenwärtigen Augenblick nicht näher äussern will, zu entscheidenden Ergebnissen führen, wird vielleicht vor unserem nächsten Kongress in das Dunkel, das die exsmdativ-lymphatische Diathese noch nmhïllt, Licht gebracht worden sein. 